\title{
X-ray Photoemission for Probing Charging/Discharging Dynamics
}

\author{
Sefik Suzer*,† and Aykutlu Dâna ${ }^{*}$ \\ Chemistry and Physics Departments, Bilkent University, 06800 Ankara, Turkey \\ Received: July 12, 2006; In Final Form: August 21, 2006
}

\begin{abstract}
A novel technique is introduced for probing charging/discharging dynamics of dielectric materials in which $\mathrm{X}$-ray photoemission data is recorded while the sample rod is subjected to $\pm 10.0 \mathrm{~V}$ square-wave pulses with varying frequencies in the range of $10^{-3}$ to $10^{3} \mathrm{~Hz}$. For a clean silicon sample, the $\mathrm{Si} 2 \mathrm{p}\left(\mathrm{Si}^{0}\right)$ peak appears at correspondingly $-10.0 \mathrm{eV}$ and $+10.0 \mathrm{eV}$ binding energy positions (20.0 eV difference) with no frequency dependence. However, the corresponding peak of the oxide $\left(\mathrm{Si}^{4+}\right)$ appears with less than $20.0 \mathrm{eV}$ difference and exhibits a strong frequency dependence due to charging of the oxide layer, which is faithfully reproduced by a theoretical model. In the simplest application of this technique, we show that the two O1s components can be assigned to $\mathrm{SiO}_{x}$ and $\mathrm{TiO}_{y}$ moeties by correlating their dynamical shifts to those of the $\mathrm{Si} 2 \mathrm{p}$ and $\mathrm{Ti} 2 \mathrm{p}$ peaks in a composite sample. Our pulsing technique turns the powerful X-ray photoemission into an even more powerful impedance spectrometer with an added advantage of chemical resolution and specificity.
\end{abstract}

Charge accumulation and dissipation (charging/discharging) in dielectric materials are vital processes for design and function of various devices and sensors. ${ }^{1}$ This is especially important for $\mathrm{SiO}_{2}$, as the thickness of the dielectric layer is expected to shrink down to few atomic layers for the next generation of metal oxide semiconductor (MOS) devices. ${ }^{2}$ Charge accumulation in the oxide layer occurs via various trapping mechanisms and is normally probed by electrical current-voltage and/or current-capacitance measurements. ${ }^{3-6}$ Photoemission utilizing UV, X-rays, and lasers has also been employed for probing very fast $\left(<10^{-9} \mathrm{~s}\right)$ charging dynamics. ${ }^{7-9}$ Core-level X-ray photoemission, XPS, is especially attractive, since additional chemical information can also be derived from the line positions of the corresponding peaks. However, the measured line positions are severely altered by local potentials developed due to the uncompensated charges resulting from photoelectron emission, especially for poorly conducting samples or regions (layers or domains) within such samples. ${ }^{10-16}$ Various methods of charge compensation have been developed using low-energy electrons, ions, and/or photons. ${ }^{17,18}$

On the other hand, one can also utilize XPS for understanding the mechanisms leading to and/or controlling of the charging/ discharging processes in materials which offer great possibilities for researchers in all fields. ${ }^{19}$ Several applications have been reported, which utilize the charging, called controlled surface charging, for extracting chemical, physical, structural, and electrical parameters of various surface species. ${ }^{20-26}$ Using a slightly different strategy and by applying voltage stress to the sample rod while recording XPS spectra, we have shown that the extent of charging can be controlled and various analytical and electrical information can be extracted. ${ }^{27-32}$ In addition to static information derived from application of dc voltage stress,

\footnotetext{
* Corresponding author (suzer@fen.bilkent.edu.tr).

Chemistry Department.

$\ddagger$ Physics Department.
}

dynamical information can also be extracted if the voltage stimuli are applied in the form of pulses, as we have recently reported..$^{30,33}$ Since an electron source cannot be turned on and off rapidly, the application of the voltage stress to the sample rod brings an important advantage for dynamical measurements.

In this contribution, we extend our investigation to analysis of XPS data recorded by applying the voltage stress in the form of bipolar square pulses for probing charging/discharging dynamics of thin dielectric surface structures. We also supplement our experimental data with a theoretical model. Previously reported dynamical photoemission measurements have been either in the ultrafast, subpicosecond regime using laser excitations $^{7-9}$ or in much longer time regimes $\left(10^{1}-10^{4}\right.$ minutes) with conventional XPS measurements. ${ }^{14,21,33-36}$ This is the first time where XPS measurements are reported using voltage pulses for probing charging/discharging dynamics of dielectric materials in the range of $10^{-3}$ to $10^{3} \mathrm{~s}$, matching those of many chemical-biochemical processes. ${ }^{37}$

The $\mathrm{SiO}_{2}$ layers were grown thermally on HF-cleaned Si(100) substrates at $600{ }^{\circ} \mathrm{C}$ in air. Thickness of the overlayer was estimated from the angular dependency of the XPS data. ${ }^{38}$ A Kratos ES300 electron spectrometer with $\mathrm{MgK} \alpha \mathrm{X}$-rays (nonmonochromatic) was used for XPS measurements. In the standard geometry, the sample accepts X-rays at $45^{\circ}$ and ejects photoelectrons at $90^{\circ}$ with respect to the surface plane. A filament placed nearby the sample provides low-energy electrons for neutralization. A typical sample is a ca. $2 \mathrm{~mm}$ silicon wafer with dimensions of $4 \times 8 \mathrm{~mm}^{2}$, electrically connected from both the top (oxide layer) and the bottom (silicon substrate) to the sample holder, which is grounded or pulsed by a square wave in the range of $10^{-3}$ to $10^{3} \mathrm{~Hz}$ while recording the spectra. For mimicking the charging behavior, a series resistor (1 Mohm) and a parallel capacitor $(0.1-1000 \mathrm{nF})$ are connected externally. The composite $\mathrm{SiO}_{x}+\mathrm{TiO}_{y}$ sample was obtained by coating the silicon substrate with a very thin layer of Ti. 

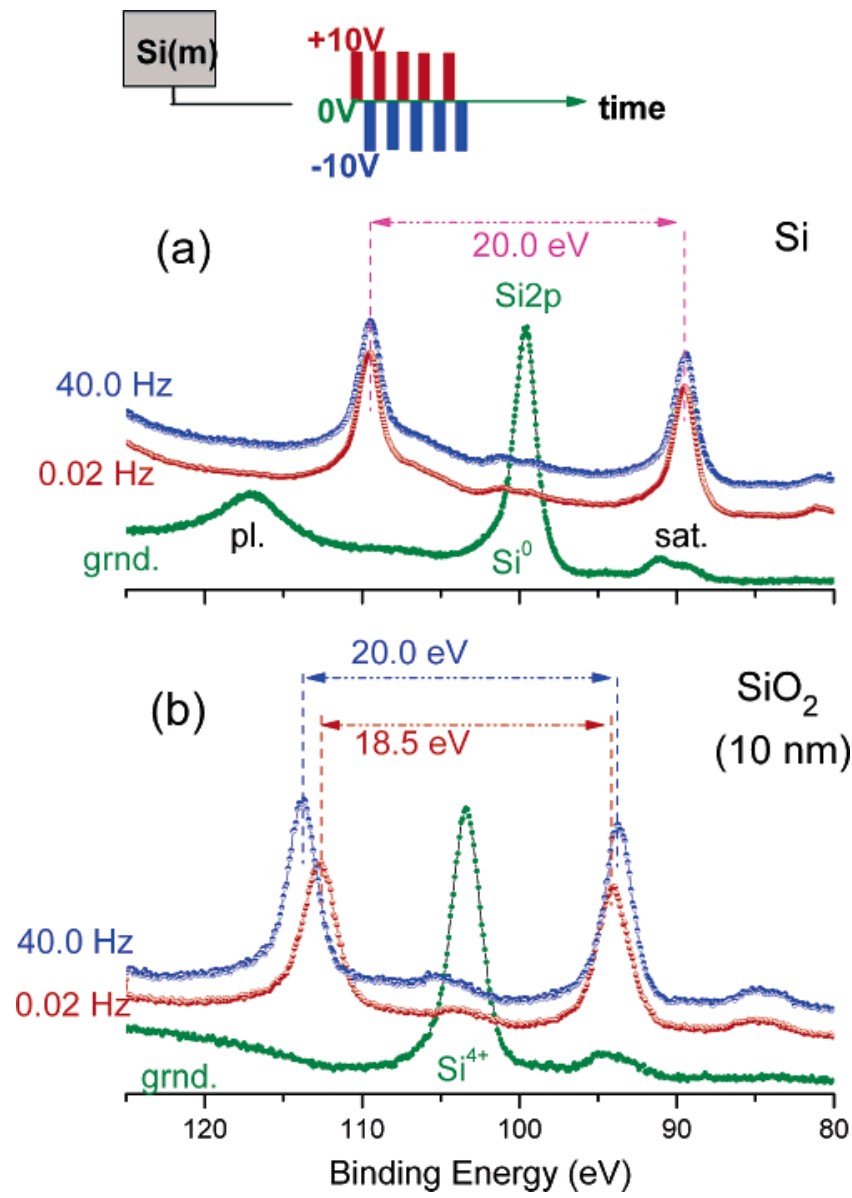

Figure 1. XPS spectrum of the Si2p region recorded when grounded (olive) and when subjected to $10.0 \mathrm{~V}$ square pulses at 0.02 (red) and 40 (blue) $\mathrm{Hz}$ frequencies: (a) a clean silicon sample and (b) a sample containing ca. $10 \mathrm{~nm}$ thermal silicon oxide layer. The square-wave modulation splits the Si2p peak into two components, and the separation between them displays a frequency dependence only for the $10 \mathrm{~nm}$ $\mathrm{SiO}_{2}$ sample. The peaks labeled as pl. and sat. correspond to the plasmon and $K \alpha_{3,4}$ satellite peaks of the Si2p, respectively. The pulsing scheme is shown in the inset.

In Figure 1a, we display the $\mathrm{Si} 2 \mathrm{p}$ region of the XPS spectra of a clean Si wafer together with another one containing ca. 10 $\mathrm{nm}$ thermal oxide layer, both recorded under voltage stress in the form of bipolar square pulses with two different frequencies. For the clean sample, the $\mathrm{Si}^{0}$ peak is split into two components appearing at correspondingly -10.0 and $+10.0 \mathrm{eV}$ with respect to its original position, since the sample spends $50 \%$ of its time at $10.0 \mathrm{~V}$ higher and lower potentials in the negative and positive cycles, respectively. For the oxide, the $\mathrm{Si}^{4+}$ peak is similarly split (as shown in Figure $1 \mathrm{~b}$ ), but the shifts are not only smaller than $10.0 \mathrm{eV}$ but also exhibit strong frequency dependence, due to dynamical charging/discharging of the oxide layer under the influence of the voltage stimuli, as was also pointed out in our previous work. ${ }^{33}$ Furthermore, the O1s peak exhibits exactly the same frequency dependence. The technique enables us to determine the impedance of different surface species.

This dynamical behavior can be experimentally simulated by introducing a simple $R C$ element tied externally to the clean $\mathrm{Si}$ sample which displays a frequency dependence as depicted in Figure 2a. In the spectrum of Si recorded at $0.1 \mathrm{~Hz}$ and without any external element, the separation between the split peaks is exactly $20.0 \mathrm{eV}$, whereas in the spectrum recorded at the same
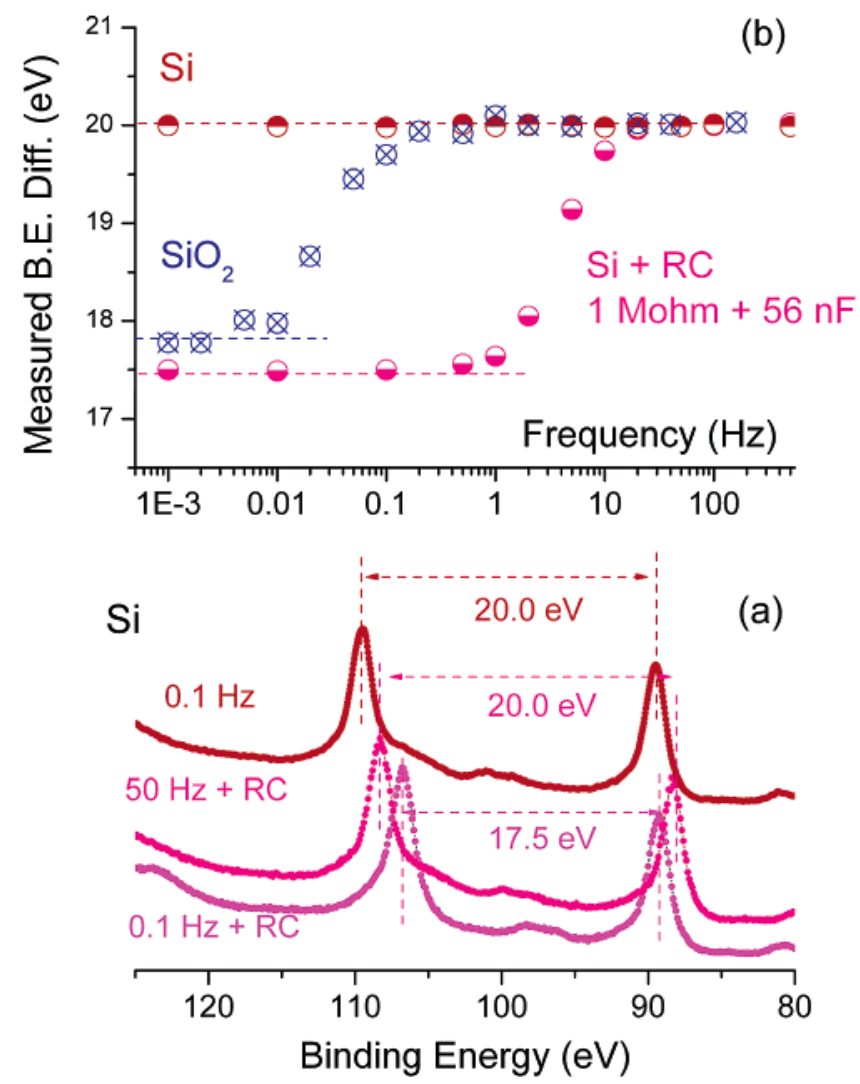

Figure 2. (a) XPS spectrum of the Si2p region of the clean silicon sample recorded at $0.1 \mathrm{~Hz}$ together with the same sample connected to the pulse generator through a $\mathrm{RC}(1 \mathrm{Mohm}+56 \mathrm{nF})$ circuit recorded at 0.1 and $50 \mathrm{~Hz}$. (b) The frequency dependence of the separation between the split peaks plotted in a semilogarithm fashion for the clean $\mathrm{Si}, 10 \mathrm{~nm} \mathrm{SiO}$, and clean $\mathrm{Si}$ tied through the RC circuit.

frequency and containing an external $R C(R=1$ Mohm, $C=$ $56 \mathrm{nF}$ ), the separation becomes as low as $17.5 \mathrm{eV}$, which recovers at higher frequencies. The frequency dependence of the separation between the split peaks is plotted in Figure $2 b$ for the $\mathrm{SiO}_{2}$, the clean $\mathrm{Si}$, and the clean $\mathrm{Si}$ tied through an external $R C$ in a semilog fashion to emphasize the remarkable resemblance between the frequency dependence of the $\mathrm{Si}+$ $R C$ to that of the $\mathrm{SiO}_{2}$. As far as the frequency dependence is concerned, the $\mathrm{SiO}_{2}$ layer acts like a simple $R C$ circuit. One can further the experimental simulation by combining the two and measuring the frequency dependence of the very same 10 nm oxide sample connected this time to an external $R C$ circuit. The experimental results are shown in Figure 3, together with our theoretical simulation results to be described below, again yielding a perfect agreement. Doubling of the S-type curve is the signature of two different charging $R C$ elements [(i) $\mathrm{SiO}_{2}$ layer, (ii) $\mathrm{Si}+R C$ ], which is exactly reproduced by our theoretical simulations.

Our XPS measurements can be modeled with a lumped circuit as shown in Figure 4a, where the electron gun and the photoelectron currents are modeled by a voltage-controlled current source (VCCS). The nonlinear voltage-current curve for the VCCS was extracted through analysis of a series of XPS measurements by applying dc voltages to the clean silicon sample in series with a known external resistor. The VCCS current-voltage dependence is assumed to be the same throughout the entire dynamic measurements. The excitation voltage source $V_{\mathrm{EX}}(t)$ can be a time-varying voltage with any arbitrary shape. To calculate the XPS spectra, we need to solve the 


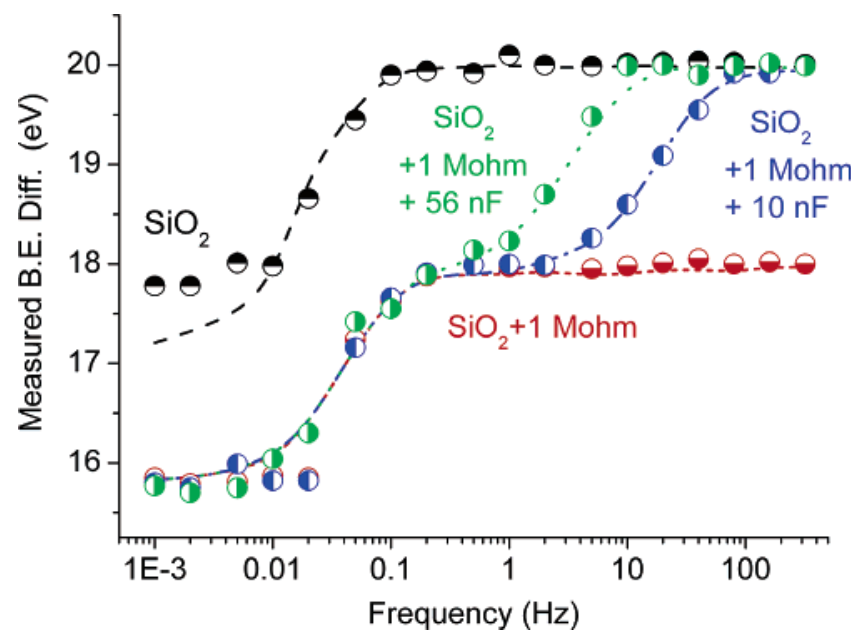

Figure 3. The frequency dependence of the separation between the split peaks plotted in a semilogarithm fashion for the $10 \mathrm{~nm} \mathrm{SiO}{ }_{2}$ only (black), tied through $1 \mathrm{Mohm}$ resistance (red), tied through $1 \mathrm{Mohm}$ resistance and $10 \mathrm{nF}$ (blue), and $56 \mathrm{nF}$ (green) capacitance. The dashed curves are results of the theoretical simulation (see text).

(a)

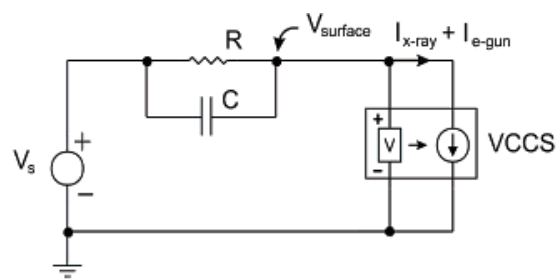

(b)
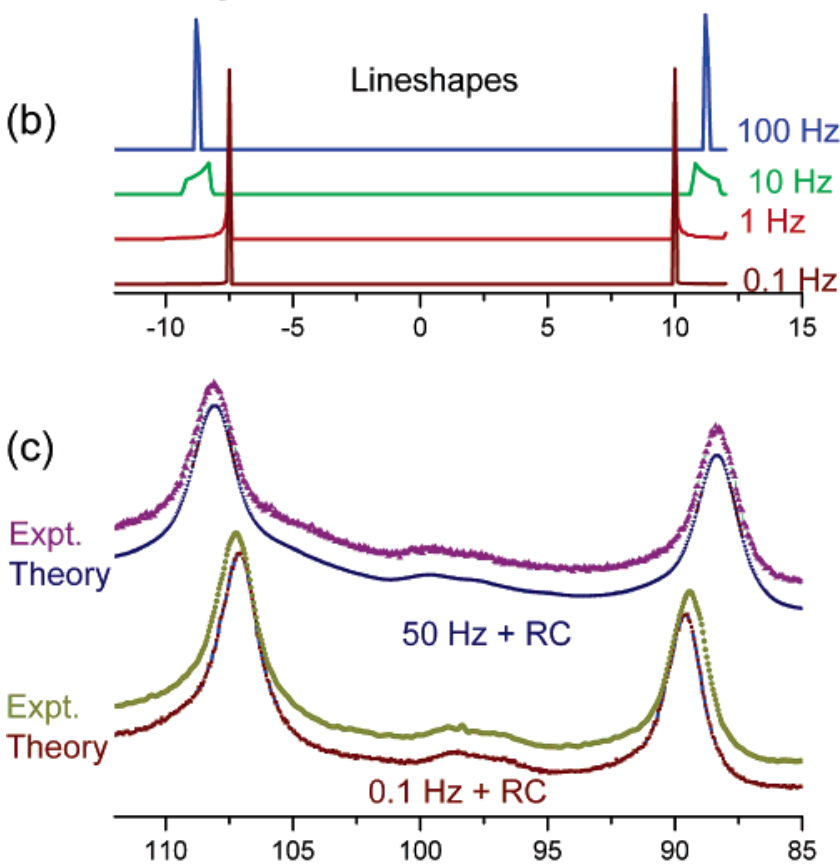

Figure 4. (a) The circuit used for simulation of the frequency dependence measured and given in Figures 2 and 3. (b) Calculated line shapes. (c). Experimental and simulated spectra of the clean $\mathrm{Si}$ tied through the $\mathrm{RC}(1 \mathrm{Mohm}+56 \mathrm{nF})$ circuit at two different frequencies.

differential equation describing the change of the surface potential $V_{\mathrm{S}}(t)$ given by

$$
C \frac{\mathrm{d}\left(V_{\mathrm{S}}-V_{\mathrm{EX}}\right)}{\mathrm{d} t}+\frac{V_{\mathrm{S}}-V_{\mathrm{EX}}}{R}+I_{\mathrm{S}}\left(V_{\mathrm{S}}\right)=0
$$

where, $R$ and $C$ are, respectively, the effective resistance and the capacitance between the surface and the source as shown in Figure $4 \mathrm{a}$, and $I_{\mathrm{S}}\left(V_{\mathrm{S}}\right)$ is the surface-potential-dependent current of the electron flood gun. Solutions can be obtained by numerical integration for arbitrary excitations $V_{\mathrm{EX}}(t)$. For a periodic excitation of period $T$, one can calculate a line-shape function that gives the intensity of the XPS signal at a voltage shift of $v$ as

$$
g(v)=\frac{1}{T} \int_{0}^{T} \delta\left[V_{\mathrm{S}}(t)-v\right] \mathrm{d} t
$$

Note that $\int_{-\infty}^{\infty} g(v) \mathrm{d} v=1$, and hence, the line-shape function is properly normalized. The XPS spectrum recorded by the spectrometer $S_{\mathrm{XPS}}(v)$ can now be calculated by convolution of the line-shape function $g(v)$ with the original spectrum $S_{0}(v)$ obtained by grounding the sample.

$$
S_{\mathrm{XPS}}(v)=\int_{-\infty}^{\infty} g(v-\lambda) S_{0}(\lambda) \mathrm{d} \lambda
$$

By numerically solving eq 1 for the values of $R=1 \mathrm{M} \Omega$ and $C=56 \mathrm{nF}$ for square-wave excitation, we calculate the lineshape functions through eq 2 as shown in Figure 4b. It can be seen that, depending only on the period of the excitation and charge-discharge time constants of the sample system, the left and right peaks of the line-shape function shift. If the excitation signal period is much higher than the sample time constants, we are working in a regime similar to dc. In this case, the peaks are shifted almost to the positions that would be obtained by applying -10 or $+10 \mathrm{~V}(\mathrm{dc})$ to the sample, respectively. Also, the broadening is almost zero. If the excitation frequency is high, the average surface potential is shifted due to the nonlinear nature of the VCCS, but the separation between the left and right peaks is closer to $20 \mathrm{~V}$. Also, there is a greater broadening of the peaks. It is seen through calculations that, for the squarewave case, the peaks have maximum broadening, as half of the period of excitation coincides with the charging/discharging time constant of the sample. The broadening for the square-wave case goes to zero as excitation frequency goes to zero or infinity. Resulting spectra for two square-wave excitations are also shown in Figure $4 c$ together with the experimental data. Agreement between calculated and measured data is again remarkable. The theory works equally well for square-wave and sinusoidal excitation voltages as tested on the same $10 \mathrm{~nm} \mathrm{\textrm {SiO } _ { 2 }}$ sample (not reproduced here), showing that it can be used to calculate the XPS spectra under any arbitrary waveform excitation.

This method measures an effective time constant comprising an effective resistance and capacitance of the films under X-rays and low-energy electron exposure. The charging and discharging of the films are nonlinearly dependent on the surface electrical field created, since they are described by tunneling or hopping processes. The simple $R C$ model can be replaced with a more accurate model that takes into account the distribution of charge traps using a distributed network. However, the ultimate model has to include E-field and position-dependent charge-discharge rates as well as local density of trap states.

The charging and discharging processes we probe are related to trapping and detrapping of the holes created in the valence band of the oxide following the very fast $\left(<10^{-12} \mathrm{~s}\right)$ photoemission process. ${ }^{21}$ Therefore, the time constants we measure are comparable to the time constants derived by time-dependent leakage currents determined for MOS systems under X-ray exposure $^{3-5}$ and/or using scanning capacitance microscopy. ${ }^{6}$

The dynamical measurement paves the way to a number of analytical tools for extracting information about charging/ discharging of surface structures. As the simplest application of this technique, we investigated a composite surface containing 


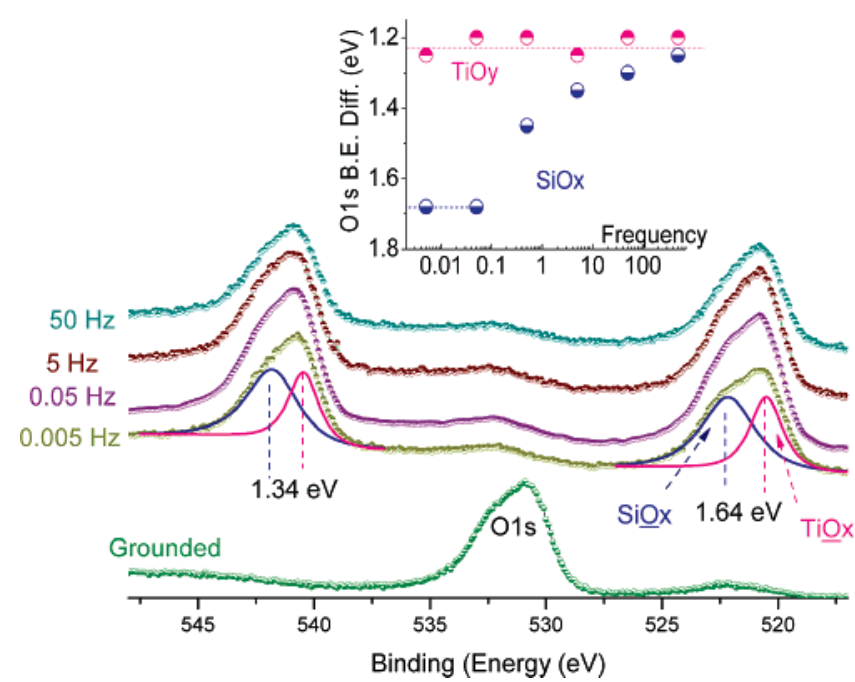

Figure 5. XPS spectrum of the O1s region of a composite sample containing both oxidized silicon $\left(\mathrm{SiO}_{x}\right)$ and titanium $\left(\mathrm{TiO}_{y}\right)$ recorded while grounded (olive) and subjected to square pulses at various frequencies. The O1s can be curve-fitted to two O1s components which display different frequency dependence as given in the insert.

both $\mathrm{SiO}_{x}$ and $\mathrm{TiO}_{y}$ on a silicon substrate. When subjected to square pulses with different frequencies, the Si2p and the Ti2p peaks display different behavior due to their different extent of charging/discharging capacity. More interestingly, the O1s peak is observed as a broad peak which can be decomposed to two components, one of which exhibits a frequency dependence and can be identified as belonging to $\mathrm{SiO}_{x}$ (same behavior as $\mathrm{Si} 2 \mathrm{p}$ ), and the other does not, which is identified as TiOy (same behavior as Ti2p), solely on their frequency dependence as shown in Figure 5. However, more work is needed to clarify the cause(s) of the different charging behavior of the two oxides.

In conclusion, we show that dynamics of the charging/ discharging of dielectric surface structures can be probed by examining the frequency dependence, upon subjecting the sample to externally applied voltage pulses while recording XPS spectra. This behavior can be experimentally simulated by a conducting sample tied externally to an $R C$ circuit. The behavior of the dielectric layer and/or the conducting sample with the external $R C$ can also be theoretically simulated using a lumped circuit where the electron gun and the photoelectron currents are modeled by a voltage-controlled current source. This technique turns XPS to a powerful and noncontact impedance spectrometer with an added advantage of chemical resolution and specificity. We expect the technique to be especially useful for analyses of fragile organic layers such as self-assembled monolayers (SAMs), and so forth, where electrical measurements are more difficult to perform.

Acknowledgment. This work was partially supported by TUBA (Turkish Academy of Sciences) and by TUBITAK (The Scientific and Technological Research Council of Turkey) through grant no. 105M094.

\section{References and Notes} 1981.

(1) Sze, S. M. Physics of Semiconductor Devices; Wiley: New York,

(2) Muller, D. A.; Sorsch, T.; Moccio, S.; Baumann, F. H.; EvansLutterodt, K.; Timp, G. Nature (London) 1999, 399, 758.

(3) Curtis, O. L., Jr.; Srour, J. R. J. Appl. Phys. 1977, 48, 3819.

(4) Hughes, R. C. Phys. Rev. B 1977, 15, 2012.

(5) Wang, T.; Cheng, T. E.; Chiang, L. P.; Wang, C. H.; Zons, N. K.; Huang, C. IEEE Trans. Electron Devices 1998, 45, 1511.

(6) Kang, C. J.; Buh, G. H.; Lee, S.; Kim, C. K.; Mang, K. M.; Im, C.; Kuk, Y. Appl. Phys. Lett. 1999, 74, 1815.

(7) Hovel, H.; Grimm, B.; Pollmann, M.; Reihl, B. Phys. Rev. Lett. 1998, 81, 4608.

(8) Marsi, M.; Belkhou, R.; Grupp, C.; Panaccionne, G.; TalebIbrahimi, A. Nahon, L.; Garzella, D.; Nutarelli, D.; Renault, E.; Roux, R.; Couprie, M. E.; Billardon, M. Phys. Rev. B 2000, 61, R5070.

(9) Bauer, M.; Lei, C.; Read, K.; Tobey, R.; Gland, J.; Murnane, M. M.; Kapteyn, H. C. Phys. Rev. Lett. 2001, 87, 025501.

(10) Countour, J. P.; Mouvier, G. Chem. Phys. Lett. 1975, 33, 237.

(11) Barr, T. L. Crit. Rev. Anal. Chem. 1991, 22, 229.

(12) Lau, W. M. J. Appl. Phys. 1989, 65, 2047.

(13) Fernandez, A.; Espinos, J. P.; Leinen, D.; Gonzales-Elipe, A. R.; Sanz, J. M. Surf. Interface Anal. 1994, 22, 111.

(14) Tielsch, B. J.; Fulghum, J. E. Surf. Interface Anal. 1997, 25, 904.

(15) Havercroft, N. J.; Sherwood, P. M. A. Surf. Interface Anal. 2000 $29,232$.

(16) Briggs, D.; Seah, M. P. Practical surface analysis. Part I. Auger and $X$-ray photoelectron spectroscopy, 2nd ed.; John Wiley \& Sons Ltd.: England, 1996.

(17) Cazaux, J. J. Electron Spectrosc. Relat. Phenom. 1999, 105, 155

(18) Cazaux, J. J. Electron Spectrosc. Relat. Phenom. 2000, 113, 15.

(19) Grimm, M.; Langer, B.; Schlemmer, S.; Lischke, T.; Becker, U.; Widdra, W.; Gerlich, D.; Flesch, R.; Ruhl, E. Phys. Rev. Lett. 2006, 96, 066801.

(20) Lau, W. M.; Wu, X. W. Surf. Sci. 1991, 245, 345.

(21) Iwata, S.; Ishizaka, A. J. Appl. Phys. 1996, 79, 6653.

(22) Doron-Mor, H.; Hatzor, A.; Vaskevich, A.; van der Boom-Moav, T.; Shanzer, A.; Rubinstein, I.; Cohen, H. Nature (London) 2000, 406, 382.

(23) Shabtai, K.; Rubinstein, I.; Cohen, S. R.; Cohen, H. J. Am. Chem. Soc. 2000, 122, 4959 .

(24) Cohen, H. Appl. Phys. Lett. 2004, 85, 1271.

(25) Dubey, M.; Gouzman, I.; Bernasek, S. L.; Schwartz, J. Langmuir 2006, 22, 4649 .

(26) Gouzman, I.; Dubey, M.; Carolus, M. D.; Schwartz, J.; Bernasek, S. L. Surf. Sci. 2006, 600, 773.

(27) Suzer, S. Anal. Chem. 2003, 75, 7026

(28) Karadas, F.; Ertas, G.; Suzer, S. J. Phys. Chem. B 2004, 108, 1515.

(29) Ertas, G.; Suzer, S. Surf. Interface Anal. 2004, 36, 619.

(30) Demirok, U. K.; Ertas, G.; Suzer, S. J. Phys. Chem. B 2004, 108, 5179.

(31) Ertas, G.; Demirok, U. K.; Suzer, S. Appl. Surf. Sci. 2005, 249, 12.

(32) Ertas, G.; Demirok, U. K.; Atalar, A.; Suzer, S. Appl. Phys. Lett. $\mathbf{2 0 0 5}, 86,183110$

(33) Karabudak, E.; Demirok, U. K.; Suzer, S. Surf. Sci. Lett 2006, 600, 2.

(34) Feldman, Y.; Zak, A.; Tenne, R.; Cohen, H. J. Vac. Sci. Technol., A 2003, 21, 1752 .

(35) Hagimoto, Y.; Fujioka, H.; Oshima, M. Appl. Phys. Lett. 2000, 77,4175 .

(36) Hirose, K.; Sakano, K.; Takahashi, K.; Hattori, T. Surf. Sci. 2002, 507-510, 906 .

(37) Bard, A. J.; Faulkner, L. R. Electrochemical Methods; Wiley: New York, 2001

(38) Mitchell, D. F.; Clark, K. B.; Bardwell, J. A.; Lennard, W. N.; Massoumi, G. R.; Mitchell, I. V. Surf. Interface Anal. 1994, 21, 44. 